\title{
Macrospin Tunneling and Magnetopolaritons with Nanomechanical Interference
}

\author{
Alexey A. Kovalev, ${ }^{1,4}$ Lorien X. Hayden, ${ }^{2}$ Gerrit E. W. Bauer, ${ }^{3,5}$ and Yaroslav Tserkovnyak ${ }^{1}$ \\ ${ }^{1}$ Department of Physics and Astronomy, University of California, Los Angeles, California 90095, USA \\ ${ }^{2}$ Department of Physics, University of Missouri, Columbia, Missouri 65211, USA \\ ${ }^{3}$ Institute for Materials Research, Tohoku University, Sendai 980-8577, Japan \\ ${ }^{4}$ Department of Physics and Astronomy, University of California, Riverside, California 92521, USA \\ ${ }^{5}$ Kavli Institute of NanoScience, Delft University of Technology, Lorentzweg 1, 2628 CJ Delft, The Netherlands
}

(Received 10 November 2010; published 4 April 2011)

\begin{abstract}
We theoretically address the quantum dynamics of a nanomechanical resonator coupled to the macrospin of a magnetic nanoparticle by both instanton and perturbative approaches. We demonstrate suppression of the tunneling between opposite magnetizations and destruction of magnetopolaritons (coherent magnetomechanical oscillations) by nanomechanical interference. The predictions can be verified experimentally by a molecular magnet attached to a nanomechanical bridge.
\end{abstract}

DOI: 10.1103/PhysRevLett.106.147203

PACS numbers: 75.80.+q, 75.45.+j, 75.50.Xx, 85.65.+h

The first direct observation of quantum behavior of a macroscopic mechanical resonator constituting a nanoelectromechanical system (NEMS) has been reported recently [1]. This opens a wide range of new possibilities for testing quantum-mechanical principles on macroscopic objects and has the potential to impact sensor technology. NEMSs have also been suggested to operate as qubits and memory elements for quantum-information processing [2]. Proposals and realizations of two-level systems (e.g., superconducting qubits) coupled to mechanical modes $[2,3]$ allow quantum measurements on the mechanical resonator. Here, we study quantum effects in a NEMS coupled to a ferromagnetic nanoparticle such as a singlemolecule magnet (SMM).

The dynamics of a magnetic order parameter and a mechanical resonator are coupled by conservation of angular momentum [4]. The magnetization dynamics of a ferromagnetic particle [4] as well as macrospin-tunneling oscillations in a SMM [5] should in principle induce magnetomechanical motion. However, the semiclassical treatment fails when the coupling becomes stronger and quantum mechanical effects such as freezing of spin tunneling [8] manifest themselves, as discussed below.

In this Letter, we consider a torsional nanomechanical resonator [see Fig. 1(a)] consisting of a load (e.g., a magnetic nanoparticle or a SMM attached to a paddle) and a mechanical link to the base (e.g., a nanotube or a chemical bond). The projection of the wave function of the macrospin on the two lowest energy levels is equivalent to a harmonic oscillator coupled to a two-level system [6]. The interference effects discussed here can be understood by considering a mechanical resonator in the $n$th excited state [see Fig. 1(b)], which has $n+1$ probability maxima at different torsion angles, e.g., the first excited state can be thought of as a superposition of two wave functions peaked at different torsion angles [7].
For the first excited state, the instanton path on a unit sphere [the middle geodesic in Fig. 1(c)] is split into two equivalent ones [geodesics with a scatter in Fig. 1(c)] due to magnetic anisotropies defining the tunneling trajectory (e.g., an easy $x y$-plane anisotropy). The area between the equivalent paths in Fig. 1(c) multiplied by the spin $S$ is equal to the difference in the phases accumulated by the two paths, which leads to a complete suppression of tunneling for a phase difference of $\pi$. Interference effects are therefore observable when $S \sqrt{\hbar / I_{x} \omega_{r}} \sim \pi$, where $I_{x}$ is the moment of inertia of the load and $\omega_{r}$ is the natural frequency of the resonator (the feasibility of this regime will be discussed below). We find that interference is most

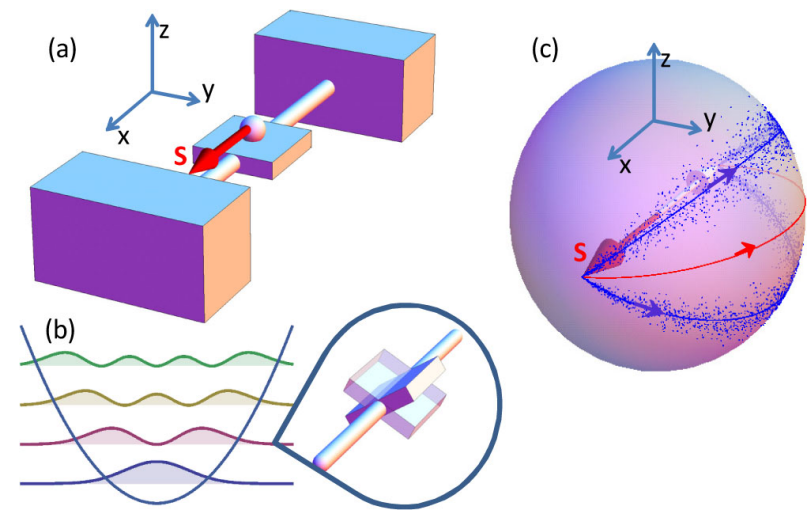

FIG. 1 (color online). (a) A torsional resonator consisting of a beam (e.g., nanotube) and a load (e.g., a magnetic nanoparticle or a SMM attached to a paddle). (b) Probability of finding the resonator at a given angle for the four lowest energy levels. (c) The first excited state of the resonator is effectively a superposition of two states with positive and negative torsion angles. The spin-reversal tunnel path on a unit sphere with a rigid resonator (middle geodesic) splits into two equivalent ones (fuzzy paths represent uncertainty in the tilt of the mechanical resonator), allowing interference. 
significant in systems in which there is a certain ratio between the spin and mechanical angular momenta which is analogous to the selection (parity) rules in large-angle macrospin tunneling [9]. We predict that the effect is rather robust and can be observed at experimentally achievable temperatures in state-of-the-art structures. Furthermore, tunneling can be suppressed by raising temperature (thus repopulating the lowest states but without increasing decoherence). Below, we derive rigorously that quantummechanical oscillations of the resonator indeed lead to a suppression of macrospin tunneling and destruction of magnetomechanical modes. Using the instanton approach, we find that the coupling of a magnetic particle with an easy-plane anisotropy to a mechanical resonator can only lower the tunneling rate, thus stabilizing the spin.

Consider a magnetic nanoparticle that behaves as a rigid, spin- $S$ object and is characterized by the magnetic anisotropy energy $\hat{H}_{A}=E\left(\hat{S}_{x}, \hat{S}_{y}, \hat{S}_{z}\right)$, where $\hat{S}_{x,(y, z)}$ are the spin-projection operators (in units of $\hbar$ ). Here, $E\left(S_{x}, S_{y}, S_{z}\right)$ is the classical magnetic energy corresponding to an easy $x$ axis and a transverse perturbation (parity symmetric about the $y-z$ plane) that couples the magnet to the torsional motion (e.g., an easy $x y$-plane anisotropy). Our complete Hamiltonian is

$$
\hat{H}=\hat{H}_{r}+e^{-i \hat{S}_{x} \hat{\varphi}} \hat{H}_{A} e^{i \hat{S}_{x} \hat{\varphi}}+\gamma \hbar \hat{S}_{x} B,
$$

where the spin is coupled to a single mechanical mode with frequency $\omega_{r}$ and Hamiltonian $\hat{H}_{r}=\hbar \omega_{r}\left(\hat{a}^{\dagger} \hat{a}+1 / 2\right)$ in terms of creation or annihilation operators $\hat{a}^{\dagger} / \hat{a}$. The second term in Eq. (1) describes magnetic anisotropy, taking into account its orientation with respect to the lattice in terms of the torsion angle $\hat{\varphi}=\left(\alpha \hat{a}^{\dagger}+\alpha^{*} \hat{a}\right) / 2 S$ $\left(\alpha=S \sqrt{2 \hbar / I_{x} \omega_{r}}\right.$ for the system in Fig. 1; however, the analytical solution presented below will also hold for an arbitrary complex $\alpha$ ). The third term is due to the external magnetic field $B$ along the $x$ axis ( $\gamma$ is minus the gyromagnetic ratio). We now transform the Hamiltonian (1) by a unitary transformation $e^{-i \hat{S}_{x} \hat{\varphi}}$ to the rest frame:

$$
\hat{H}_{R}=\hbar \omega_{r}\left(\hat{\tilde{a}}^{\dagger} \hat{\tilde{a}}+1 / 2\right)+\hat{H}_{A}+\gamma \hbar \hat{S}_{x} B .
$$

Here, $\hat{\tilde{a}}=\hat{a}-i \hat{S}_{x} \alpha / 2 S$. We describe tunneling between low-lying states of the macrospin by path integrals in which coherent states are constructed using the Heisenberg-Weyl (the resonator) and $\mathrm{SU}(2)$ (the spin) groups from a state $|\uparrow, n\rangle$ by a standard procedure [10]:

$$
|\boldsymbol{\Omega}, z\rangle=e^{z \hat{a}^{\dagger}-z^{*} \hat{a}} e^{-i \hat{S}_{z} \phi} e^{-i \hat{S}_{y} \theta}|\uparrow, n\rangle .
$$

Index $n$ here stands for a Fock state with $n$ phonons in the mechanical mode, $\uparrow$ refers to a macrospin state pointing to the north pole, $\theta$ and $\phi$ are the Euler angles defining direction $\boldsymbol{\Omega}=(\theta, \phi)$ of the macrospin, and the complexvalued $z=z_{r}+i z_{i}$ parametrizes a generalized coherent state of the harmonic oscillator.

In the large- $S$ limit, the transition amplitude between two approximate eigenstates at $\theta=\pi / 2, \quad \phi=0$, $z=i \alpha / 2$ and $\theta=\pi / 2, \phi=\pi, z=-i \alpha / 2$ can be expressed through coherent-state path integrals [11] as

$\left\langle-\mathbf{x},-i \alpha / 2\left|e^{-i \int d t \hat{H}_{R}}\right| \mathbf{x}, i \alpha / 2\right\rangle=\int \mathcal{D} \Omega e^{i\left(\mathcal{S}_{k}+\mathcal{S}_{E}\right) / \hbar}$,

where $\mathcal{D} \Omega \sim \prod_{t} d \phi_{t} d\left(\cos \theta_{t}\right) d z_{t} d z_{t}^{*}, \mathcal{S}_{k}=\hbar \int d t\left[\left(\dot{z}_{r} z_{i}-\right.\right.$ $\left.\left.\dot{z}_{i} z_{r}\right)-S \dot{\phi}(1-\cos \theta)\right]$ is the kinetic, and $\mathcal{S}_{E}=-\int d t \tilde{E}$, where $\tilde{E}=\hbar \omega_{r}\left[n+1 / 2+\left(z-i s_{x} \alpha / 2\right)\left(z^{*}+i s_{x} \alpha / 2\right)\right]+$ $E(\theta, \phi)$, the potential-energy contribution to the action in the absence of a magnetic field $\left(s_{x}=\sin \theta \cos \phi\right)$. The term $\mathcal{S}_{k}$ describes interference effects that can be exposed by treating the transition amplitude in Eq. (4) by a saddlepoint approximation. Each saddle-point path acquires a phase $S A+2 A^{\prime}$, where $A$ is the solid angle spanned by the spin paths connected to the north pole by geodesics and $A^{\prime}$ is the area enclosed by the torsional trajectories connected to the origin in the complex plane. These phases cause interference effects in the tunneling of spins [11].

We calculate the tunneling rate by a quasiclassical treatment in imaginary time. The quasiclassical equations of motion in real time minimize the action in Eq. (4) [12]:

$$
\begin{gathered}
\dot{z}_{i}=-\omega_{r} z_{r}, \quad \dot{z}_{r}=\omega_{r} z_{i}-\left(\alpha \omega_{r} / 2\right) \cos \phi \sin \theta, \\
S \dot{\theta} \sin \theta=\partial \tilde{E} / \partial \phi, \quad S \dot{\phi} \sin \theta=-\partial \tilde{E} / \partial \theta .
\end{gathered}
$$

In order to find the instanton path in the presence of the coupling to the mechanical mode, we integrate these equations numerically in imaginary time $(t=-i \tau)$. The splitting of the degenerate modes by the tunnel interaction can be expressed as $\Delta_{n} \propto \mathcal{C} e^{\operatorname{Re}\left(\mathcal{S}_{0}^{\mathrm{cl}}\right) / \hbar}$, where $\mathcal{S}_{0}^{\mathrm{cl}}=i \mathcal{S}_{k}+\mathcal{S}_{E}$ is the Wick rotated instanton action of a quasiclassical trajectory found by solving Eq. (5) for $\theta, \phi, z_{i}$, and $z_{r}$. $\mathcal{C}$ describes the interference of different spin trajectories under tunneling selection rules [e.g., for an easy $x y$-plane anisotropy $\mathcal{C}=\cos (\pi S)]$ [11]. For an easy-axis and easyplane anisotropy described by $E=K_{1} \cos ^{2} \theta+K_{2} \sin ^{2} \theta$ $\sin ^{2} \phi\left(K_{1}>K_{2}>0\right)$, we find that all paths are contained between two extremal paths denoted in Fig. 2(b) by dotted lines. The tunnel splitting changes from $\exp \left(-\alpha^{2} / 2\right) \Delta_{0}$ [rectangular-shaped dotted path in Fig. 2(b)] to $\Delta_{0}$ [cosineshaped dotted path in Fig. 2(b)] as we go from the limit $\omega_{r} \ll \omega_{0} \sim t_{I}^{-1}$ (which is the focus of this Letter) to the
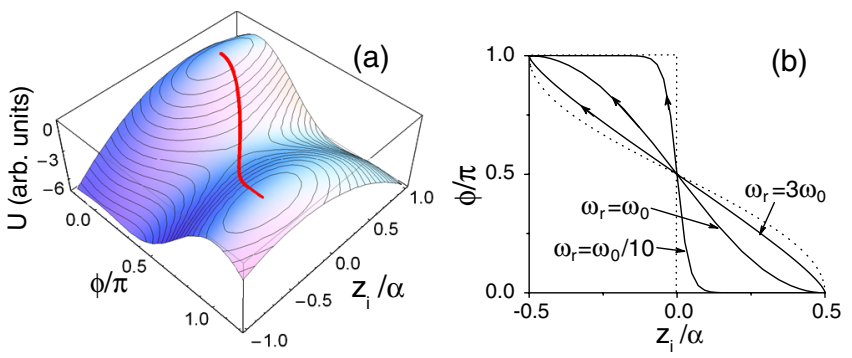

FIG. 2 (color online). (a) A particle falls in a camelbackshaped potential following the quasiclassical path (bold curve). (b) Results of a numerical integration of Eq. (5) for different frequencies of the mechanical resonator. 
limit $\omega_{r} \gg \omega_{0} \sim t_{I}^{-1}$, where $\Delta_{0}$ is the tunnel splitting without coupling to the resonator. $\omega_{0}=\sqrt{K_{1} K_{2}} / S$ and $t_{I}$ is the uncoupled (magnetic) instanton tunneling time. Such behavior can be understood by eliminating $\theta$ and $z_{r}$ from Eq. (5) and the imaginary-time Lagrangian:

$$
L=M_{1}(\phi) \dot{\phi}^{2} / 2+M_{2} \dot{z}_{i}^{2} / 2-U(\phi, z) .
$$

Here $M_{1}(\phi)=\omega_{0}^{-1}\left(1 / \sqrt{\lambda}-\sqrt{\lambda} \sin ^{2} \phi+\alpha \cos \phi \omega_{r} / 2 \omega_{0}\right)$, $M_{2}=2 / \omega_{r}, U(\phi, z)=-\sqrt{\lambda} \omega_{0} \sin ^{2} \phi-\omega_{r}\left(z_{i}-\cos \phi \alpha / 2\right)^{2}$, $\lambda=K_{2} / K_{1}$, and $\theta \approx \pi / 2$ has been used. By inspecting the motion of a particle with anisotropic mass in the potential $U(\phi, z)$ [Fig. 2(a)], we see that the quasiclassical path connecting two potential-energy maxima leads to a smaller tunnel splitting because it experiences a higher tunneling barrier compared to an uncoupled instanton (this is also expected for other magnetic anisotropies).

We show below that the quasiclassical approach only works well for $n=0$ but does not capture nanomechanical interference effects. The latter can be obtained by calculating the quantum fluctuations and by retaining the second order terms (depending on the Fock number $n$ ) in the kinetic energy of the Lagrangian. The rest of this Letter addresses the limit $t_{I} \ll \omega_{r}^{-1}$ in which the kinetic-energy contribution to the action suppresses the resonator dynamics. The resonator contribution can then be calculated by taking the matrix element between the initial and final states of the mechanical subsystem:

$$
\left\langle-\mathbf{x},-i \alpha / 2\left|e^{-i \int d t \hat{H}_{R}}\right| \mathbf{x}, i \alpha / 2\right\rangle=\kappa_{n n} \int \mathcal{D} \Omega e^{i \mathcal{S}_{I} / \hbar} .
$$

Here, $\kappa_{n m}=\left\langle n\left|e^{-i 2 S \hat{\varphi}}\right| m\right\rangle$ is the Fock states' matrix element of the displacement operator and $e^{i \mathcal{S}_{I} / \hbar}$ corresponds to the bare instanton contribution to the path integral (without the coupling to the resonator). The extra factor in the instanton contribution reflects the phases accumulated by multiple paths of the macrospin tunneling within the laboratory frame in Eq. (1). These paths destructively interfere, suppressing tunneling at specific values of $\alpha$ [see Fig. 3(a)], as becomes clear from the expression [13]:

$$
\kappa_{n m}=e^{-|\alpha|^{2} / 2}(-i \alpha)^{n-m} \sqrt{m ! / n !} L_{m}^{(n-m)}\left(|\alpha|^{2}\right),
$$

where $n \geq m$ ( $n<m$ can be obtained by complex conjugation) and $L_{m}^{(n-m)}$ is a generalized Laguerre polynomial. We can then anticipate that the tunnel splitting should be renormalized by the nanoresonator according to

$$
\Delta_{n}=\left|\kappa_{n n}\right| \Delta_{0} \leq \Delta_{0},
$$

where $\Delta_{0}$ is the tunnel splitting for a bare macrospin. A more rigorous derivation also applicable to the formation of magnetopolaritons $(m \neq n)$ is discussed below.

To tackle the resonant coupling between Fock states with arbitrary numbers of phonons, we project Hamiltonian (2) onto the basis formed by the two lowest energy states of $\hat{H}_{A}$, which is possible when the transverse perturbations are small (i.e., when $\Delta_{0} \ll \omega_{0}$-the distance
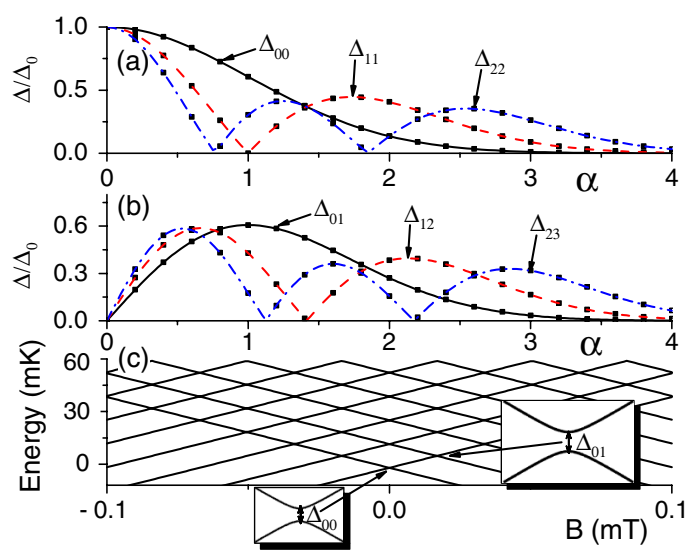

FIG. 3 (color online). (a) Tunnel splittings as a function of the macrospin-resonator coupling $\alpha$ for the first three excited states of the resonator. The curves show analytical results, while the squares are based on the numerical diagonalization of the Hamiltonian corresponding to an $\mathrm{Fe}_{8}$ SMM. (b) Analogous plot for tunnel splittings of the magnetopolariton modes corresponding to the Fock states differing by one phonon. (c) Lowest energy levels of the $\mathrm{Fe}_{8} \mathrm{SMM}$ coupled to a mechanical resonator obtained by numerical diagonalization. $\Delta_{0} / \hbar \omega_{r}=3 \times 10^{-6}$ (the energy is offset by $-28.239 \mathrm{~K}$ ).

to the third energy state). We can represent the ground and first excited states split by $\Delta_{0}$ as [8] $\Psi_{ \pm}=\left(\left|\psi_{\mathbf{x}}\right\rangle \pm\right.$ $\left.\left|\psi_{-\mathbf{x}}\right\rangle\right) / \sqrt{2}$, where states $\left|\psi_{ \pm_{\mathbf{x}}}\right\rangle$ represent perturbations of $| \pm \mathbf{x}\rangle$. The leading-order projection procedure, $\hat{H}_{p}=$ $\sum_{\mu, \nu= \pm \mathbf{x}}\left\langle\psi_{\mu}|\hat{H}| \psi_{\nu}\right\rangle\left|\psi_{\mu}\right\rangle\left\langle\psi_{\nu}\right|$, leads to [8]

$$
\hat{H}_{p}=\left[\begin{array}{cc}
\hat{H}_{r}+\gamma \hbar S B & -e^{-i 2 S \hat{\varphi}} \Delta_{0} / 2 \\
-e^{i 2 S \hat{\varphi}} \Delta_{0} / 2 & \hat{H}_{r}-\gamma \hbar S B
\end{array}\right],
$$

where we use $\left\langle\psi_{m}\left|\hat{S}_{x}\right| \psi_{n}\right\rangle \cong \pm S \delta_{m n}$ for states $\left|\psi_{ \pm \mathbf{x}}\right\rangle$. In Eq. (10) we treat $\Delta_{0}$ as a small perturbation. Therefore, in the vicinity of the resonant magnetic field corresponding to the crossing of Fock states $m$ and $n$, we can further project Eq. (10) onto the states $m$ and $n$ :

$$
\hat{H}_{n m}=\left[\begin{array}{cc}
E_{n} & -\kappa_{n m} \Delta_{0} / 2 \\
-\kappa_{n m}^{*} \Delta_{0} / 2 & E_{m}
\end{array}\right]
$$

arriving at eigenenergies $E_{n m}=\left(E_{n}+E_{m}\right) / 2 \pm$ $\sqrt{\left(E_{n}-E_{m}\right)^{2}+\Delta_{n m}^{2}} / 2$, where the energy of the Fock state $n(m)$ is $E_{n}=\hbar \omega_{r}(n+1 / 2)+\gamma \hbar S B\left[E_{m}=\hbar \omega_{r}(m+\right.$ $1 / 2)-\gamma \hbar S B]$ and $\Delta_{m n}=\left|\kappa_{m n}\right| \Delta_{0}$. We can immediately see that the magnetopolariton and tunnel splittings are given by $\Delta_{m n}$, which is the main result of this Letter.

We confirm our analytical results by an exact diagonalization of Hamiltonian (1) in the basis of 30 Fock states for spin $S=10$, which corresponds to a $630 \times 630$ matrix. A typical spin Hamiltonian describing a SMM reads

$$
\hat{H}_{A}=-D \hat{S}_{x}^{2}+E\left(\hat{S}_{z}^{2}-\hat{S}_{y}^{2}\right)+C\left(\hat{S}_{+}^{4}+\hat{S}_{-}^{4}\right),
$$

where $S_{ \pm}=S_{z} \pm i S_{y}$. The anisotropy constants correspond to an $\mathrm{Fe}_{8} \mathrm{SMM}$ with $D=0.292 \mathrm{~K}, E=0.046 \mathrm{~K}$, 
and $C=-2.9 \times 10^{-5} \mathrm{~K}[9]$. Such parameters result in the tunnel splitting $\Delta_{0} / k_{B}=4.5 \times 10^{-8} \mathrm{~K}$ in the absence of coupling to the resonator. The resonator frequency is chosen as $\hbar \omega_{r} / k_{B}=3 \times 10^{5} \Delta_{0} / k_{B} \sim 15 \mathrm{mK}$, where $k_{B}$ is the Boltzmann constant. In Fig. 3(c), we plot the calculated lowest eigenenergies. The energy (anti)crossings at zero field corresponding to the tunnel splitting are shown in Fig. 3(a) as a function of the coupling parameter $\alpha$. We observe a perfect agreement between the results of Eq. (9) (lines) and the results of the numerical diagonalization (squares). At specific values of $\alpha$, we observe destructive interference that completely quenches the macrospin tunneling. The tunnel splittings in Fig. 3(c) at nonzero magnetic fields correspond to magnetopolariton formation and are only possible for a finite coupling to the resonator. The magnetopolariton splittings reveal interference effects as a function of this coupling [see Fig. 3(b) for analytical (lines) as well as numerical (squares) results].

The tunnel splittings can be measured by the LandauZener method employed in Ref. [9]. The mechanical resonator has to be cooled to temperatures $T \ll \hbar \omega_{r} / k_{B}$ (thus higher frequency resonators are preferable), e.g., by quantum-optical [14] or cryogenic techniques (in Ref. [1] $\left.\hbar \omega_{r} / k_{B} \sim 0.1 \mathrm{~K}\right)$. The critical parameter for the observation of magnetopolariton modes and interference effects is the spin-resonator coupling $\alpha$. We estimate its value for a device that contains a SMM strongly absorbed to a paddle of the size $20 \times 20 \times 10 \mathrm{~nm}^{3}$, with a singlewall carbon nanotube serving as a mechanical link [see Fig. 1(a)]. For a torsional spring constant $K=10^{-18} \mathrm{~N} \cdot \mathrm{m}$ [15], $S=37$ (for a $\mathrm{Mn}_{17} \mathrm{SMM}$ [16]), and a moment of inertia $I_{x}=10^{-36} \mathrm{~kg} \cdot \mathrm{m}^{2}$, we obtain $\omega_{r}=\sqrt{K / I_{x}} \sim$ $1 \mathrm{GHz}$ and $\alpha \sim 0.02$. This coupling is large enough for the observation of magnetopolariton modes but too small for observing interference effects for which $\alpha$ has to be comparable to 0.5 [see Fig. 3(a)]. Sufficiently large values of the coupling can be achieved in a $\mathrm{Mn}_{12} \mathrm{SMM}$ bridged between leads [17]. For parameters similar to the ones used in Ref. [5], $I_{x} \sim 10^{-41} \mathrm{~kg} \cdot \mathrm{m}^{2}, \omega_{r} \sim 1 \mathrm{GHz}$, and $S=10$, we arrive at $\alpha \sim 1.5$. The spin-resonator coupling can be increased by lowering the torsional stiffness and the moment of inertia or by increasing the spin $S$. The energy levels in Fig. 3(c) can be used for quantum manipulations and single-phonon control of a mechanical resonator. This requires larger tunnel splittings in order to overcome decoherence. The tunnel splitting can be increased by applying the magnetic field normal to the anisotropy axis [18].

To conclude, we found a quantum-mechanical solution for the coupled motion of a macrospin and a mechanical resonator. We study tunnel splittings and avoided level crossings corresponding to formation of magnetopolaritons, both of which should be detectable by quantumoptical techniques or by studying Landau-Zener transitions [9]. In the strong spin-resonator coupling regime, we predict suppression of the tunneling of magnetization and destruction of magnetopolaritons by interference of the spin tunneling paths resulting from the quantum state of the resonator. We predict that the magnetism in SMMs can be significantly stabilized against quantum fluctuations by sticking them to mechanical resonators with large quantum fluctuations. Results presented here are relevant for possible realizations of quantum control of magnetization at a single-phonon level.

We thank DARPA, Alfred P. Sloan Foundation, NSF-DMR-0840965 (Y. T.) and NSF-PHY-0850501 (L. H.), and the Dutch FOM foundation (G. B.).

[1] O. D. O'Connell et al., Nature (London) 464, 697 (2010).

[2] A. N. Cleland and M. R. Geller, Phys. Rev. Lett. 93, 070501 (2004); M. R. Geller and A. N. Cleland, Phys. Rev. A 71, 032311 (2005); S. Savel'ev, X. Hu, and F. Nori, New J. Phys. 8, 105 (2006).

[3] J. Hauss et al., New J. Phys. 10, 095018 (2008); A. D. Armour and M.P. Blencowe, New J. Phys. 10, 095004 (2008); M. D. LaHaye et al., Nature (London) 459, 960 (2009).

[4] A. A. Kovalev, G. E. W. Bauer, and A. Brataas, Appl. Phys. Lett. 83, 1584 (2003); Phys. Rev. Lett. 94, 167201 (2005); Phys. Rev. B 75, 014430 (2007).

[5] R. Jaafar and E. M. Chudnovsky, Phys. Rev. Lett. 102, 227202 (2009); R. Jaafar, E. M. Chudnovsky, and D. A. Garanin, Europhys. Lett. 89, 27001 (2010).

[6] E. Jaynes and F. Cummings, Proc. IEEE 51, 89 (1963); E. K. Irish et al., Phys. Rev. B 72, 195410 (2005).

[7] The ground state has one maximum smeared by quantum fluctuations, which makes it difficult to observe complete suppression of tunneling by interference, i.e., tunneling is gradually suppressed as fluctuations become stronger.

[8] E. M. Chudnovsky and D. A. Garanin, Phys. Rev. B 81, 214423 (2010).

[9] W. Wernsdorfer et al., J. Appl. Phys. 87, 5481 (2000).

[10] A. Perelomov, Generalized Coherent States and Their Applications (Springer, New York, 1986).

[11] E. M. Chudnovsky and L. Gunther, Phys. Rev. Lett. 60, 661 (1988); D. Loss, D. P. DiVincenzo, and G. Grinstein, Phys. Rev. Lett. 69, 3232 (1992); M. N. Leuenberger and D. Loss, Phys. Rev. B 63, 054414 (2001).

[12] By eliminating $z_{i}$ these equations correspond to Lagrangian $\mathcal{L}=I \dot{\varphi}^{2} / 2-K \varphi^{2} / 2+S \hbar \dot{\phi} \cos \theta+S \hbar \dot{\varphi} \sin \theta \cos \varphi-E$ in which $\varphi=\alpha z_{r} / S$. This Lagrangian is derived in M.F. O'Keeffe and E.M. Chudnovsky, arXiv:1011.3134v1 [Phys. Rev. B (to be published)] without the term $-K \varphi^{2} / 2$.

[13] K. E. Cahill and R. J. Glauber, Phys. Rev. 177, 1857 (1969).

[14] T. Rocheleau et al., Nature (London) 463, 72 (2009).

[15] J. C. Meyer, M. Paillet, and S. Roth, Science 309, 1539 (2005).

[16] E. E. Moushi et al., Inorg. Chem. 48, 5049 (2009).

[17] S. Barraza-Lopez et al., Phys. Rev. Lett. 102, 246801 (2009).

[18] E. del Barco et al., Europhys. Lett. 47, 722 (1999). 